山्山FARAÇAISE

$\gg \mathrm{DE}$

댈.EAGOGIE

\section{Revue française de pédagogie}

Recherches en éducation

203 | 2018

Les pratiques éducatives des parents enseignants

\title{
Les pratiques éducatives familiales des enseignants : des parents comme les autres?
}

Teachers' educational practices at home: Parents like the others?

Agnès van Zanten

\section{(2) OpenEdition \\ 1 Journals}

Édition électronique

URL : https://journals.openedition.org/rfp/8076

DOI : $10.4000 /$ rfp.8076

ISSN : 2105-2913

Éditeur

ENS Éditions

Édition imprimée

Date de publication : 30 juin 2018

Pagination : 5-14

ISBN : 979-10-362-0190-5

ISSN : 0556-7807

Référence électronique

Agnès van Zanten, «Les pratiques éducatives familiales des enseignants : des parents comme les autres? ", Revue française de pédagogie [En ligne], 203 | 2018, mis en ligne le 30 juin 2018, consulté le 04 janvier 2023. URL : http://journals.openedition.org/rfp/8076 ; DOI : https://doi.org/10.4000/rfp. 8076 


\title{
Les pratiques éducatives familiales des enseignants : des parents comme les autres?
}

\author{
Agnès van Zanten
}

Ce dossier s'intéresse aux pratiques de suivi de la scolarité de leurs enfants par un groupe socioprofessionnel, celui des enseignants. Nous y avons réuni quatre articles présentant et discutant des apports nouveaux dans ce domaine. Le choix d'un tel sujet peut surprendre : est-il vraiment utile de se pencher sur les modes éducatifs de ce groupe professionnel et, plus largement, de chaque groupe professionnel? Repère-t-on ainsi autre chose que des légères variations autour de régularités déjà largement documentées, liées à l'appartenance à des classes sociales plus larges? Et même si l'étude des enseignants fait apparaître des traits singuliers de ce groupe, cela renouvelle-t-il notre regard sur la relation entre les familles et l'école ou, de façon plus ambitieuse encore, sur le fonctionnement du système éducatif dans son ensemble? Notre réponse affirmative à ces questions s'appuie sur trois séries d'arguments que nous développons brièvement dans cette introduction.

\section{Analyser à la loupe le «métier de parent»}

Un premier argument en faveur de l'intérêt d'un tel sujet est le surcroît d'intelligibilité sur l'évolution et le contenu des pratiques d'accompagnement de la scolarité que l'on obtient en s'intéressant à un groupe particulièrement investi dans ce domaine et dont l'action dans la sphère domestique revêt un caractère quasi professionnel. Documenter les efforts pédagogiques de ses membres permet de donner alors tout son sens à une notion qui s'est largement imposée au cours des dernières décennies mais qui a souvent été utilisée de façon métaphorique, celle d'un «métier de parent». L'émergence de cette notion est liée au constat, tant par les acteurs sociaux eux-mêmes que par les chercheurs, d'une intensification de l'accompagnement éducatif parental (Sayer, Bianchi \& Robinson, 2004). Ce processus est lié à des transformations structurelles et culturelles de l'institution familiale comme de l'institution scolaire. Du côté de la famille, il est important d'évoquer en premier lieu le lien entre l'élévation générale du niveau d'études des nouvelles générations, et celui des femmes en particulier, et la propension à s'investir dans l'éducation des enfants (Chamboredon \& Prévot, 1973). Les parents sont par ailleurs d'autant plus portés à consacrer du temps à ces derniers que les fratries sont moins nombreuses qu'autrefois et beaucoup plus souvent le résultat d'un choix consciemment assumé. Les enfants sont également beaucoup plus 
valorisés dans les sociétés contemporaines en tant que vecteurs de la reproduction ou de l'amélioration de la position sociale des parents mais aussi en tant que sources de bonheur et d'épanouissement (Zélizer, 1985; de Singly, 2009; Déchaux, 2014), ce qui rend le travail éducatif parental plus crucial, complexe et noble que par le passé. À cela il faut ajouter la tendance, renforcée par la profusion de recherches sur les compétences enfantines, à attribuer aux enfants toujours plus de capacités de perception, de raisonnement ou de créativité, ainsi que l'émergence d'un ensemble d'outils et de services marchands (livres, guides, coachs...) visant à orienter et à soutenir les parents dans leur rôle éducatif (Hays, 1996).

L'importance que prennent l'anticipation et l'accompagnement de la scolarité au sein des pratiques parentales est quant à elle liée aux évolutions de l'école et de son rôle social. L'allongement des scolarités a entraîné l'allongement parallèle du suivi parental des parcours scolaires au point que l'on constate aujourd'hui son extension jusqu'à la poursuite des études des enfants dans l'enseignement supérieur (van Zanten, 2015; Hamilton, 2016). La diversification et la hiérarchisation croissantes de ces parcours et les possibilités de choix offertes par des systèmes d'enseignement plus complexes et formellement plus ouverts qu'autrefois favorisent par ailleurs une forte concurrence parentale pour accéder aux plus convoités d'entre eux (van Zanten, 2019). Ceci va de pair avec le développement de stratégies de recueil et de tri des informations et des conseils, et de négociation avec, et de pression sur, les professionnels de l'enseignement (Ball \& Vincent, 1998; Lareau \& McCrory Calarco, 2012). Enfin, l'investissement parental s'explique aussi par la centralité des diplômes dans l'insertion professionnelle doublée de la nécessité croissante des diplômés de se distinguer sur le marché de l'emploi en mettant en avant d'autres qualités distinctives qui excèdent celles que le diplôme est censé certifier (Brown, 2000). Si ces transformations concernent tous les parents, les parents enseignants sont parmi ceux qui ont développé les formes les plus sophistiquées d'adaptation à ces nouveaux cadres sociaux. L'analyse aussi bien statistique que qualitative de leurs pratiques discrédite tout d'abord définitivement l'idée que le rôle des familles dans la réussite scolaire des enfants concernerait prioritairement la transmission «osmotique » d'un certain nombre de savoirs, savoir-faire et savoir-être (Garcia, 2018). Cette transmission continue certes de enfance autour notamment de l'acquisition du langage et d'un rapport spécifique à celui-ci (Bernstein, 1977; Bourdieu \& Passeron, 1970), mais aussi tout au long de l'enfance et de l'adolescence. Elle favorise en particulier l'incorporation d'un certain nombre de dispositions aujourd'hui très valorisées sous le terme de compétences sociales ou «soft skills» s'avérant utiles pour les jeunes dans l'interaction aussi bien avec les enseignants (McCrory Calarco, 2018) qu'avec de futurs employeurs (Brown \& Hesketh, 2004).

Comme le montrent tous les articles de ce dossier, les parents enseignants s'investissent aussi de façon beaucoup plus visible et systématique dans le suivi de la scolarité. Accompagnant pour beaucoup de façon quotidienne leurs enfants dans la réalisation des devoirs à la maison, ces parents échangent aussi régulièrement avec les enseignants, participent aux réunions et aux sorties et sont nombreux à faire partie des associations des parents et des instances comme les conseils de classe ou d'établissement. Comme le souligne notamment l'article de Fanny Salane et Muriel Letrait, ces parents exercent aussi un contrôle étroit de leurs enfants hors de l'espace scolaire par le recours massif à des activités encadrées. La fréquentation de celles-ci favorise la maîtrise de connaissances et compétences culturelles diverses qui, sans avoir nécessairement des effets directs sur la réussite scolaire, participent à l'acquisition d'une «culture générale » encore hautement valorisée dans l'enseignement secondaire et supérieur français mais aussi de compétences organisationnelles et de dispositions à la rationalisation et à la rentabilisation du temps libre très utiles à la poursuite des études (Coulangeon, 2018).

Ces parents peuvent de ce fait apparaître comme des parents «modèles» répondant en tout point aux attentes des institutions d'enseignement. Un regard plus approfondi, comme celui que propose l'article de Séverine Kakpo et Patrick Rayou, montre cependant que l'efficacité de leurs pratiques ne repose ni sur une soumission aveugle à ces attentes, ni sur la mobilisation à la maison des mêmes pratiques qu'ils mettent en œuvre auprès de leurs propres élèves dans leur activité professionnelle. En effet, d'une part, l'appropriation des attentes scolaires se traduit plutôt par des formes de surenchère par rapport aux exigences de leurs collègues et par des modes de colonisation de l'espace scolaire évoqués plus en détail plus loin. Et, d'autre part, les enseignants font preuve d'autres compétences pédagogiques à la maison que celles qu'ils mobilisent dans la salle de classe en donnant notamment une 
large place à des dimensions expressives généralement absentes des institutions scolaires françaises. Comme chez d'autres parents des classes supérieures mais probablement de façon plus habile, leur investissement d'ordre émotionnel dans l'éducation de leurs enfants vise à préserver une relation affective étroite avec eux, la prise en compte de leurs goûts et besoins et l'harmonie familiale (Reay, 2000) mais, en articulant soutien affectif, apports cognitifs et pression sociale, cette «intimité enrichie» (Stefansen \& Aarseth, 2011) s'avère aussi un formidable outil pédagogique réservé à la sphère domestique et à leur seule progéniture.

\section{Enrichir le débat sur la reproduction des classes sociales}

Un deuxième argument plaidant en faveur de l'étude des pratiques éducatives familiales des enseignants est l'intérêt d'un tel sujet pour l'analyse des processus de reproduction sociale. On sait aujourd'hui qu'une part très significative de l'influence de l'origine sociale sur la position sociale des individus s'exerce via le niveau d'études atteint par ces derniers, autrement dit que l'éducation formelle est un des principaux facteurs de la reproduction intergénérationnelle du statut social (Vallet, 2017). Même si d'autres facteurs contribuent aussi à expliquer les différences interindividuelles des niveaux d'éducation, l'analyse des pratiques éducatives orientées vers la réussite scolaire des différents groupes sociaux revêt donc une importance centrale dans l'étude de la reproduction et de la mobilité sociales. La question se pose néanmoins de savoir quel est le niveau d'analyse le plus pertinent pour cerner précisément la transmission familiale et sociale d'avantages scolaires. Plus précisément, par rapport au thème de ce dossier, est-il plus approprié d'analyser les pratiques éducatives des enseignants en les considérant comme un groupe à part ou comme un groupe appartenant à des ensembles plus vastes, à savoir à des classes ou des fractions de classes?

Une telle question ne peut être explorée sans tenir compte des catégories utilisées par la statistique publique française pour classer les différents groupes sociaux que mobilisent les trois dernières études de ce dossier. Deux nomenclatures sont particulièrement utilisées. La première, et la plus répandue, est celle des "professions et catégories professionnelles» (PCS) de I'Insee mobilisée dans de nombreuses enquêtes pour mesurer les liens entre les origines sociales d'un côté, et les pratiques ou les trajectoires sociales dans différents domaines, dont l'éducation, de l'autre. Elle comprend, au niveau le plus agrégé, six postes pour les personnes en activité (agriculteurs; artisans, commerçants et chefs d'entreprise; cadres et professions intellectuelles supérieures; professions intermédiaires; employés; ouvriers). Les enseignants y sont ventilés entre deux postes : les professeurs du secondaire et du supérieur sont placés dans la catégorie «cadres et professions intellectuelles supérieures; les instituteurs dans celle des "professions intermédiaires». Cette distinction s'explique par le fait que la nomenclature PCS, dès sa conception très hybride, tient compte du statut des actifs, de leur métier, de leur qualification et de leur place dans la hiérarchie professionnelle mais aussi d'un ensemble de pratiques sociales (fécondité, loisirs, opinions politiques...). La prise en compte de ces pratiques lors de la première refonte des PCS en 1982 a été fortement inspirée par la vision bourdieusienne de l'espace social dans laquelle les professeurs et les instituteurs, bien que situés sur un même axe horizontal du côté des «fractions intellectuelles», occupent des places différentes sur l'axe vertical, en lien avec leur détention de volumes inégaux de capital économique et culturel et les effets de ces combinaisons sur leurs pratiques culturelles (Amossé, 2013; Bourdieu, 1979). La deuxième est celle de la DEPP qui regroupe ces mêmes catégories en quatre ensembles plus larges ( "favorisée $A$ », «favorisée $B$ », «moyenne» et « défavorisée ») en extrayant certains postes des catégories agrégées de l'Insee, notamment les instituteurs qui sont rattachés à la première catégorie, sur la base d'une construction privilégiant le critère de la distance à l'école' (voir annexe).

Les trois articles de ce dossier qui se fondent sur des enquêtes mobilisant ces nomenclatures ou celle du niveau des diplômes apportent des éclairages convergents ou complémentaires sur la spécificité du groupe enseignant. Concernant les trajectoires scolaires, trois résultats essentiels, déjà mis en avant par les chercheurs de la DEPP et de l'Insee (Caille \& O'Prey, 2005; Sautory, 2007), sont avancés et discutés. Le premier concerne la très grande similitude des parcours scolaires des enfants d'enseignants et des enfants d'autres groupes faisant partie de la catégorie «cadres et professions intellectuelles supérieures», y compris, si l'on se centre exclusivement sur la réussite et les types de

1 Comme l'indique T. Rocher (2016), les critères de regroupement utilisés dans cette nomenclature n'ont néanmoins pas été explicités lors de sa création. 
trajectoires jusqu'au baccalauréat, des enfants des professeurs des écoles, ce qui semble justifier les regroupements opérés par I'Insee mais aussi par la DEPP. Le second, c'est que cette comparaison fait néanmoins apparaître des écarts, certes faibles mais toujours à la faveur des enfants d'enseignants, ce qui plaide pour porter sur eux, et sur les pratiques éducatives de leurs parents, un regard particulier. Le troisième, que souligne notamment ici Géraldine Farges, concerne la différenciation «horizontale » qui s'opère à l'issue du baccalauréat. On constate en effet que les enfants des professeurs du secondaire - mais pas ceux des professeurs des écoles -, qui gardent un léger avantage sur les autres membres de la catégorie «cadres et professions intellectuelles supérieures » en termes de nombre d'années d'études post-baccalauréat, accèdent aussi souvent qu'eux aux filières sélectives de l'enseignement supérieur. Ils se distinguent néanmoins par le fait d'opter pour des filières «académiques» menant à des carrières comme l'enseignement ou la recherche moins bien rémunérées que celles (études de médecine, écoles d'ingénieurs et de commerce) choisies par les enfants d'autres membres des classes supérieures. Ces différences renvoient à celles entre les fractions intellectuelles et économiques des classes supérieures finement étudiées par Bourdieu (1979) sur lesquelles nous revenons plus loin, celles-ci recoupant largement à leur tour celles entre professions supérieures du public et du privé soulignées par d'autres auteurs (de Singly \& Thélot, 1988; Hugrée, Penissat \& Spire, 2015).

À ces différences de trajectoires correspondent des différences qualitatives dans les pratiques éducatives parentales. Comme le montre notamment Annie Lasne, si l'investissement éducatif des parents enseignants dans la scolarité de leurs enfants apparaît dans une large mesure comparable à celui d'autres parents relevant de la catégorie «cadres et professions intellectuelles», les premiers pratiquent de façon plus intense et systématique une véritable colonisation de l'école en étant particulièrement attentifs à écarter tous les obstacles, à mettre à profit toutes les opportunités pour favoriser l'excellence scolaire de leurs enfants et pour assurer leur maintien dans la voie royale menant aux études supérieures les plus académiquement prestigieuses. Les seconds sont en revanche davantage attachés à la préservation d'un entre-soi et d'un environnement scolaires favorables certes à l'acquisition par leurs enfants d'un capital scolaire, mais aussi d'un capital social et de valeurs leur permettant de s'insérer dans I'univers des classes supérieures.
Ces trois articles soulignent aussi la spécificité des ressources dont disposent les parents enseignants pour favoriser la scolarité de leurs enfants, notamment de deux d'entre elles. La première est leur capital culturel. Ce dernier se distingue de celui des autres membres de la catégorie "cadres et professions intellectuelles supérieures » moins par son volume, bien que le niveau d'éducation des enseignants français soit à la fois plus élevé et plus homogène que celui des cadres par exemple, que par des aspects plus qualitatifs concernant notamment l'accès à des informations sur l'école que leur procure leur insertion institutionnelle et les compétences pédagogiques associées à l'exercice de leur métier. La seconde est le temps. En effet, la synchronie entre leurs horaires et temps de travail contraints et ceux des établissements fréquentés par leurs enfants et la possibilité d'organiser leur propre temps de travail non contraint de façon à se rendre disponibles pour aider ces derniers à faire leurs devoirs ou pour les accompagner à diverses activités culturelles constituent un avantage majeur par rapport à d'autres catégories sociales (Letrait \& Salane, 2015).

Faut-il alors considérer les enseignants comme une «micro classe» (Weeden \& Grusky, 2005), c'est-à-dire comme un groupe professionnel doté de traits suffisamment singuliers pour qu'il mérite d'être classé à part? Pour cela, il faudrait pouvoir mettre en évidence, d'une part, l'existence d'une forte homogénéité de ce groupe et, d'autre part, celle de pratiques et de caractéristiques fortement distinctives dans plusieurs domaines. Or, si d'autres recherches seraient nécessaires pour étayer notamment cette dernière dimension, les travaux récents qui leur ont été consacrés soulignent plutôt, d'une part, l'hétérogénéité du groupe du point de vue d'un ensemble de traits (parcours sociaux et de formation, revenus, conditions de travail, pratiques culturelles et sociabilité, rapport au politique) et, d'autre part, la proximité, notamment du sousgroupe des enseignants du secondaire, avec d'autres groupes sociaux du point de vue aussi bien du choix du conjoint que du rapport à la culture légitime (Farges, 2017). D'ailleurs, l'article de Géraldine Farges pointe les différences qui se maintiennent entre le corps des enseignants du primaire et du secondaire malgré l'élévation de l'origine sociale des premiers (Charles \& Cibois, 2010). Il souligne aussi l'efficacité en termes de stratégies scolaires des couples composés d'un cadre ou membre d'une profession intellectuelle supérieure et d'un enseignant du secondaire, couples dont les enfants ont de très fortes chances d'intégrer les filières 
et établissements d'élite de l'enseignement supérieur (Euriat \& Thélot, 1995; Albouy \& Wanecq, 2003).

II faut ajouter à cela que les pratiques éducatives des parents enseignants sont très proches de celles de l'ensemble social que nous avons appelé les « intellectuels», se distinguant en revanche nettement de celles des «technocrates». Ces deux groupes, qui correspondent grosso modo aux deux fractions des classes supérieures qu'oppose Pierre Bourdieu dans La Distinction (1979), privilégient des choix scolaires et des méthodes éducatives contrastés qui reflètent le volume et le type de ressources dont ils disposent ainsi que les valeurs et les compétences de leurs membres en lien avec leur position sociale mais aussi avec la nature de leur activité professionnelle (van Zanten, 2009a, 2009b). D'autres travaux mobilisant cette typologie ont, depuis, montré sa pertinence pour étudier différents aspects de la socialisation familiale comme les choix vestimentaires ou celui des activités culturelles ou sportives des enfants (Menesson \& Julhe, 2012; Court \& Menesson, 2015). Ils ont aussi montré qu'au sein de filières sélectives comme les instituts d'études politiques (IEP) accueillant des étudiants issus de ces deux fractions de classe, ceux ayant des parents «intellectuels», dont font partie dans ce schéma les enfants d'enseignants, s'investissent davantage dans les études et optent pour des masters tournés vers le secteur public et des professions à dominante intellectuelle, sociale et humanitaire. En revanche, ceux ayant des parents "technocrates» choisissent principalement des masters dans les domaines de la finance, du conseil ou du marketing et privilégient au sein de l'institution des pratiques associatives favorisant leur insertion dans ces univers (Lozach, 2018).

\section{Revisiter l'étude des inégalités d'éducation}

En raison de ces dernières considérations, nous estimons qu'il est plus pertinent d'étudier les enseignants non pas comme une classe en soi mais comme une composante essentielle de la fraction intellectuelle des classes supérieures dont ils ont historiquement contribué à dessiner les contours et à asseoir la place dans la société française (Charle, 1988). Un troisième argument nous conduit néanmoins à continuer à plaider en faveur de l'étude systématique de leurs pratiques éducatives ainsi qu'en faveur de la mise en relation de leurs pratiques en tant que professionnels et en tant que parents. Il s'agit de l'intérêt heuristique que cette analyse conjointe présente du point de vue de la compréhension des effets sur les inégalités scolaires de l'interaction entre les logiques institutionnelles et les logiques familiales. Cette mise en relation permet notamment de mieux comprendre l'importance que les enseignants en tant que groupe social et que groupe professionnel attachent à la production et à la reconnaissance de l'excellence scolaire, et comment les pratiques qui découlent de cet attachement structurent les dynamiques de compétition et de parrainage (Turner, 1960) au cœur de la re-production des inégalités sociales par le système éducatif français (van Zanten, 2016).

Pour comprendre l'attachement des enseignants à l'excellence scolaire, il est important de rappeler une évidence : le groupe socioprofessionnel des enseignants est celui dont aussi bien la position sociale que la légitimité professionnelle de ses membres dépendent le plus étroitement de la certification de leur valeur par l'école. On se saurait donc pas s'étonner de constater que le souci de maintenir leur position sociale supérieure en même temps que l'emprise qu'ils possèdent sur les destinées d'autres groupes sociaux, via la licence et le mandat associés à leur activité professionnelle (Hugues, 1996), les conduisent à accorder une très grande importance aux trajectoires et aux diplômes scolaires. Cette focalisation sur la scolarisation va de pair avec une forte adhésion à l'idéologie méritocratique, c'est-à-dire au fait que le déroulement de ces parcours et les titres qui les sanctionnent reposent sur des évaluations des talents et des efforts personnels et non sur des avantages liés à la naissance ou à l'argent (Tenret, 2011). Cette valorisation du mérite scolaire va néanmoins de pair avec le souci de réserver les cheminements et les grades ou titres les plus prestigieux à un petit groupe d'élus afin de préserver leur valeur distinctive, intrinsèque et extrinsèque, dans la mesure où cette valeur est perçue comme étroitement liée à celle des profils et au nombre des individus reconnus comme «méritants».

Ce souci se traduit dans l'activité professionnelle des enseignants par l'importance accordée aux outils et mécanismes permettant d'opérer des différenciations entre les élèves «méritants» et les autres. Un des principaux est la notation, qui est dans le système éducatif français particulièrement précoce, sévère et discriminante, des niveaux d'acquisition des élèves (Merle, 2007). La façon dont cet outil a été manié a eu, jusqu'à une période récente, un effet majeur et durable 
sur le ralentissement des carrières scolaires via le mécanisme du redoublement, dont les effets paraissent dans l'ensemble négatifs bien que faisant objets de débats au sein de la communauté scientifique (Draelants, 2018). La notation et ses effets sur les carrières scolaires sont aussi étroitement liés à un autre mécanisme contribuant dans une large mesure à la ségrégation des groupes sociaux à l'école, à savoir l'orientation des élèves dans des parcours d'inégal prestige (Duru-Bellat \& Mingat, 1998; Chauvel, 2015; Cayouette-Remblière, 2016).

Ces processus jouent un rôle central dans une compétition scolaire de plus en plus violente et visible au fur et à mesure que les élèves s'approchent des transitions scolaires ayant les effets les plus puissants sur leur trajectoire : celle du collège au lycée, qui reste déterminante pour les élèves de milieu populaire, et celle du lycée vers l'enseignement supérieur qui est aujourd'hui l'horizon de presque $90 \%$ des bacheliers. Conscients de l'importance de cette compétition, les parents des classes supérieures déploient tous des stratégies précoces et durables pour permettre à leurs enfants d'en sortir gagnants, à commencer par la transmission d'aspirations élevées et d'un sense of entitlement (Lareau, 2011) indispensables pour se projeter de façon confiante dans un avenir scolaire et professionnel ambitieux.

On constate néanmoins des différences significatives entre les parents "technocrates» et «intellectuels». Privilégiant un rapport utilitariste au savoir, les premiers ont davantage tendance à considérer l'excellence scolaire comme un moyen d'atteindre l'excellence professionnelle et sociale. Cette vision, couplée aux ressources économiques considérables dont ils disposent, les conduit, d'une part, à favoriser chez leurs enfants la recherche des stratégies les plus efficaces d'adaptation à l'institution et, d'autre part, à exercer une emprise extérieure sur les jugements scolaires par le recours à des aides payantes comme les cours particuliers, les séjours linguistiques à l'étranger ou, de façon plus marginale, le coaching en matière d'orientation. En revanche, les parents «intellectuels», et parmi eux notamment les enseignants, s'ils ne s'interdisent pas des stratégies de ce type, parient davantage sur l'entraînement des enfants à la compétition en vue d'une excellence scolaire perçue également comme un moyen de reproduction ou d'ascension sociale mais aussi comme un moyen de légitimer leur position en témoignant de leur profonde adhésion aux valeurs du système d'enseignement. Comme le montrent notam- ment dans ce dossier les articles de Séverine Kakpo et Patrick Rayou et d'Annie Lasne, ces derniers se situent dans une posture d'intériorité et non d'extériorité visà-vis des jugements scolaires. Ils s'efforcent en effet $d$ 'inculquer à leurs enfants des valeurs et des habitudes comme l'ascétisme et la régularité dans l'effort et des compétences intellectuelles comme l'autonomie, l'esprit critique et la capacité d'argumentation fortement valorisées par les enseignants et les institutions d'enseignement.

Le système scolaire se caractérise toutefois aussi par un «parrainage » des bons élèves s'articulant étroitement avec la compétition scolaire. Les gagnants aux différentes épreuves bénéficient d'un fort parrainage institutionnel dont la forme la plus visible est l'accès à des classes, des options, des filières et des établissements permettant les parcours scolaires les plus prestigieux. Parallèlement, au fur et à mesure que les élèves avancent dans le système, les épreuves les plus valorisées ne sont proposées qu'aux élèves déjà présélectionnés dans ces filières, l'exemple le plus visible et emblématique étant les concours d'accès aux grandes écoles qui ne sont en pratique que très rarement tentés par des candidats n'ayant pas été admis à suivre des classes préparatoires à ces écoles, les plus prestigieux de ces concours n'étant réussis que par les élèves ayant suivi des classes préparatoires «étoilées »² (van Zanten, 2016; Berkouk \& François, 2018). Parce qu'il récompense formellement, comme la compétition, la valeur scolaire des élèves et ne leur paraît pas incompatible avec l'idéal d'égalité de chances qu'ils épousent par ailleurs dans la mesure où ce dernier est défini comme la possibilité pour chaque élève d'aller aussi loin que lui permettent ses capacités et ses efforts (Savidan, 2007), ce parrainage est largement soutenu par les enseignants (Rauscher, 2019). C'est même le cas d'un nombre significatif de ceux exerçant dans des filières et des établissements stigmatisés favorables à l'idée de proposer un «parrainage compensatoire » aux bons élèves issus de ces contextes (van Zanten, 2010).

Or les effets de relégation et d'exclusion que produit ce «parrainage institutionnel» sur les élèves qui n'en bénéficient pas sont redoublés par le «parrainage familial » qu'il engendre en retour et auquel participent très

2 À la fin de leur première année, les meilleurs élèves des classes préparatoires scientifiques peuvent rejoindre une classe «étoilée» dont le programme d'études correspond en tous points à celui des concours d'admission des grandes écoles les plus prestigieuses. Ces classes n'existent que dans un nombre réduit de lycées d'élite. 
activement les classes supérieures du pôle économique et du pôle intellectuel, mais à nouveau sous des modalités différentes. En effet, pour avoir accès aux parcours d'élite les premiers optent souvent pour des établissements d'enseignement privés dont la sélectivité scolaire, mais aussi sociale, et l'organisation interne des activités est davantage en phase avec leurs buts et leurs conceptions. Sans écarter cette option, les seconds optent à leur tour pour une "privatisation » du système d'enseignement public grâce, comme le soulignent notamment les textes d'Annie Lasne et de Géraldine Farges, à une attention soutenue portée aux variations des conditions de scolarisation entre les établissements publics et dans leur sein, ainsi qu'aux critères de hiérarchisation de ces environnements éducatifs par leurs collègues et par les institutions d'enseignement.

\section{Conclusion}

Pour poursuivre les pistes d'analyse ouvertes dans cette introduction et dans les articles qui composent ce dossier, deux voies semblent particulièrement prometteuses. La première, que nous n'avons pas abordée dans ces pages mais dont le lecteur trouvera la trace dans les différents textes, concerne l'intérêt de mobiliser l'analyse des pratiques éducatives des enseignants pour mieux comprendre les différences dans les apports des hommes et des femmes, et plus généralement des deux membres des couples, à la scolarité des enfants, et leur complémentarité. La plus grande réussite des enfants dont la mère est enseignante par rapport à ceux dont c'est le père qui exerce cette activité témoigne de la plus grande rentabilité, à niveau scolaire parental égal, du capital maternel, ce qui est clairement la conséquence du plus grand investissement des femmes dans le suivi scolaire des enfants, mais probablement aussi

\section{Bibliographie}

ALBOUY V. \& WANECQT. (2003). «Les inégalités sociales d'accès aux grandes écoles ». Économie et Statistique, $\mathrm{n}^{\circ}$ 361, p. 27-52.

AMOSSÉ T. (2013). «La nomenclature socio-professionnelle: une histoire revisitée ». Annales. Histoire, Sciences sociales, vol. 68, n 4 , p. 1039-1075.

BALL S. J. \& VINCENT C. (1998). «"I heard it on the grapevine": "Hot" knowledge and school choice». British Journal of Sociology of Education, vol. 1, n³, p.377-400.

BERKOUK N. \& FRANÇOIS P. (2018). «Les concours sont-ils neutres? Concurrence et parrainage dans l'accès à l'École polytechnique». Sociologie, vol.9, n², p.169-196. de leur capacité à mieux mobiliser ce capital dans le domaine familial. Il s'agit là d'une question intéressante à creuser. Par ailleurs, le fait que ce sont les enfants des couples dont un membre, généralement la mère, est enseignant et un autre, le plus souvent le père, est cadre qui ont accès aux filières les plus prestigieuses de l'enseignement supérieur invite quant à lui à ne pas se focaliser exclusivement sur le capital scolaire maternel ou paternel en négligeant l'apport parental d'autres types de ressources culturelles, économiques et sociales à une scolarité réussie.

La deuxième piste concerne la possibilité de généraliser à d'autres contextes nationaux les interprétations proposées ici. S'il faut conduire des enquêtes comparatives précises pour pouvoir statuer sur ce point, on peut néanmoins, à ce stade, avancer l'hypothèse que l'efficacité scolaire des pratiques éducatives des parents enseignants dont fait état ce dossier pour la France varie fortement entre les pays. Trois critères devraient jouer un rôle important dans ces variations : tout d'abord la structure sociale de chaque pays, tant du point de vue de la différenciation verticale qu'horizontale des classes et fractions de classe; ensuite, les caractéristiques de la profession enseignante, notamment l'origine sociale, le niveau d'éducation, les revenus et les pratiques de sociabilité de ses membres; enfin, l'organisation d'ensemble du système d'enseignement et plus particulièrement la place et les modalités qu'y prennent les dynamiques de compétition et de parrainage.

Agnès van Zanten

SciencesPo, Observatoire sociologique du changement, Laboratoire interdisciplinaire d'évaluation des politiques publiques agnes.vanzanten@sciencespo.fr

BERNSTEIN B. (1977). Class, Codes and Control. Vol. 3. Londres : Routledge \& Kegan Paul.

BOURDIEU P. (1979). La Distinction. Critique sociale du jugement. Paris : Éd. de Minuit.

BOURDIEU P. \& PASSERON J.-C. (1970). La Reproduction. Éléments pour une théorie du système d'enseignent. Paris : Éd. de Minuit.

BROWN P. (2000). «The Globalization of positional competition ». Sociology, vol.34, n4, p.633-653.

BROWN P. \& HESKETH A. (2004). The Mismanagement of talent. Employability and jobs in the Knowledge Economy. Oxford : Oxford University Press. 
CAILLE J.-P. \& O'PREY S. (2005). «Estime de soi et réussite scolaire sept ans après l'entrée en sixième ». Éducation et Formations, $\mathrm{n}^{\circ} 72$, p. 25-52.

CAYOUETTE-REMBLIÈRE J. (2016). L'école qui classe. 350 élèves du primaire au bac. Paris : PUF.

CHAMBOREDON J.-C. \& PRÉVOT J. (1973). «Le métier d'enfant : définitions sociales de la prime enfance et fonctions différentielles de l'école maternelle». Revue française de sociologie, vol.14, n³, p.295-335.

CHARLE C. (1988). Les élites de la République, 1880-1900. Paris : Fayard

CHARLES F. \& CIBOIS P. (2010). «L'évolution de l'origine sociale des enseignants du primaire sur la longue durée : retour sur une question controversée». Sociétés contemporaines, vol. $77, \mathrm{n}^{\circ} 1$, p. 31-55.

CHAUVEL S. (2015). «Processus d'orientation en fin de collège : le principe d'autonomie face à deux modèles d'évaluation des élèves ». In M.-H. Jacques (dir.), Transitions en contexte scolaire. Rennes: Presses universitaires de Rennes, p.179-187.

COULANGEON P. (2018). «The impact of participation in extracurricular activities on school achievement of French middle school students: Human capital and cultural capital ». Social Forces, vol. 97, n¹, p.55-89.

COURT M. \& MENESSON C. (2015). «Les vêtements des garçons. Goûts et dégoûts parentaux au sein des classes moyennes». Terrains \& Travaux, vol.27, n², p.41-58.

DÉCHAUX J.-H. (2014). «Le sacré de l'enfant ». Revue française de sociologie, vol.55, $\mathrm{n}^{\circ} 3$, p.537-561.

DRAELANTS H. (2018). "Le redoublement est-il vraiment moins efficace que la promotion automatique? Une évidence à réinterroger ». Les Cahiers de recherche du GIRSEF, $\mathrm{n}^{\circ} 113$.

DURU-BELLAT M. \& MINGAT A. (1988). «Le déroulement de la scolarité au collège : le contexte "fait des différences"». Revue française de sociologie, vol.29, n4, p.649-666.

EURIAT M. \&THÉLOT C. (1995). «Le recrutement social de l'élite scolaire en France. Évolution des inégalités de 1950 à 1990 ». Revue française de sociologie, vol.36, n³, p.403-438.

FARGES G. (2017). Les mondes enseignants. Paris : PUF.

GARCIA S. (2018). Le goût de l'effort. La construction familiale des dispositions scolaires. Paris : PUF.

HAMILTON L. T. (2016). Parenting to a degree. How family matters for college women's success. Chicago : University of Chicago Press.

HAYS S. (1996). The cultural contradictions of motherhood. New Haven : Yale University Press.

HUGRÉE C., PENISSAT É. \& SPIRE A. (2015). «Les différences entre salariés du public et du privé après le tournant managérial des États en Europe». Revue française de sociologie, vol.56, $\mathrm{n}^{\circ} 1$, p. 47-73.

HUGUES E. (1996). Le regard sociologique. Essais choisis. Paris : éd. de l'EHESS.

LAREAU A. (2011). Unequal Childhoods. Class, race, and family life. Berkeley : University of California Press, $2^{e}$ éd.
LAREAU A. \& MCCRORY CALARCO J. (2012). «Class, cultural capital, and institutions: The case of families and schools». In S. Friske \& M. Hazel (dir.), Facing Social Class. New York : Russell Sage Foundation.

LETRAIT M. \& SALANE F. (2015). «Le temps consacré aux enfants : les enseignantes et enseignants se distinguent-ils des autres diplômés de l'enseignement supérieur? ". Économie et Statistique, $\mathrm{n}^{\circ}$ 478-479-480, p. 243-271.

LOZACH U. (2018). Approcher le pouvoir. Sociologie de l'action éducative des Instituts d'études politiques de province (19452018). Thèse de doctorat, sciences de l'éducation, université de Strasbourg.

MCCRORY CALARCO J. (2018). Negociating opportunities. How the middle class secures advantages in school. Oxford: Oxford University Press.

MENESSON C. \& JULHE S. (2012). «L'art (tout) contre le sport : la socialisation culturelle des enfants des milieux favorisés ». Politix, vol. 99, n³, p.109-128.

MERLE P. (2007). Les notes. Secrets de fabrication. Paris : PUF.

RAUSCHER J.-B. (à paraître 2019). Des enseignants d'élite? Sociologie des professeurs de classe préparatoire. Paris : Éd. du Cerf.

REAY D. (2000). «A useful extension of Bourdieu's conceptual framework? Emotional capital as a way of understanding mothers' involvement in their children's education». Sociological Review, vol.48, p.568-585.

ROCHER T. (2016). "Construction d'un indice de position sociale des élèves ", Éducation \& formations, n 90, p. 5-27.

SAUTORY O. (2007). «La démocratisation de l'enseignement supérieur : évolution comparée des caractéristiques sociodémographiques des bacheliers et des étudiants ». Éducation \& formations, $n^{\circ} 74$, p. 49-64.

SAVIDAN P. (2007). Repenser l'égalité des chances. Paris : Grasset.

SAYER L., BIANCHI S. \& ROBINSON J. (2004). «Are parents investing less in children? Trends in mothers' and fathers' time with children ». American Journal of Sociology, vol.110, $\mathrm{n}^{\circ} 1$, p.1-43.

SINGLY F. (de) (2009). Comment aider l'enfant à devenir luimême? Paris : Armand Colin.

SINGLY F. (de) \& THÉLOT C. (1988). Gens du public, gens du privé. La grande différence. Paris : Bordas.

STEFANSEN K. \& AARSETH H. (2011). «Enriching intimacy: The role of the emotional in the "resourcing" of middle-class children ». British Journal of Sociology of Education, vol.32, $\mathrm{n}^{\circ} 3$, p.389-405.

TENRET É. (2011). L'école et la méritocratie. Représentations sociales et socialisation scolaire. Paris : PUF.

TURNER R. H. (1960). «Sponsored and contest mobility and the school system ", American Sociological Review, vol.25, $\mathrm{n}^{\circ} 6, \mathrm{p} .855-867$.

VALLET L.-A. (2017). «Mobilité sociale entre générations et fluidité sociale en France. Le rôle de l'éducation». Revue de l'OFCE, $\mathrm{n}^{\circ} 150$, p. 27-67.

WEEDEN K. A \& GRUSKY D. B. (2005). «The case for a new 
class map ». American Journal of Sociology, vol.111, $\mathrm{n}^{\circ} 1$, p. 141-212.

van ZANTEN A. (2009a). "Le travail éducatif parental dans les classes moyennes et supérieures : deux modes contrastés d'encadrement des pratiques et des choix des enfants ». Informations sociales, vol.4, n 154, p. 80-87.

van ZANTEN A. (2009b). Choisir son école. Stratégies familiales et médiations locales. Paris : PUF.

van ZANTEN A. (2010) "L'ouverture sociale des grandes écoles : diversification des élites ou renouveau des politiques publiques d'éducation?». Sociétés contemporaines vol.78, p.69-96.

van ZANTEN A. (2015). «A family affair: Reproducing elite positions and preserving the ideals of meritocratic competition and youth autonomy ». In A. van Zanten, S. J. Ball
\& B. Darchy-Koechlin (dir.), World Yearbook of Education 2015. Elites, privilege and excellence: The national and global redefinition of educational advantage. Londres \& New York: Routledge, p.29-42.

van ZANTEN A. (2016). «La fabrication familiale et scolaire des élites et les voies de mobilité ascendante en France ». L'Année sociologique, vol.66, n¹, p. 81-114.

van ZANTEN A. (2019). «Neo-liberal influences in a "conservative" regime: The role of institutions, family strategies and market devices in access to higher education in France». Comparative education, vol. $55, \mathrm{n}^{\circ} 2$. En ligne: <https://www.tandfonline.com/doi/full/10.1080/0305006 8.2019.1619330> (consulté le 9 juin 2019).

ZÉLIZER V. (1985). Pricing the priceless child. The changing social value of children. New York : Basic Books. 


\section{Annexe}

Tableau 1. Les professions et catégories professionnelles (PCS) de I'Insee Postes concernant la population active

\begin{tabular}{|c|c|}
\hline $\begin{array}{l}\text { PCS en } 8 \text { postes } \\
\text { ( } 6 \text { pour la population active) }\end{array}$ & $\begin{array}{l}\text { PCS en } 24 \text { postes } \\
\text { ( } 18 \text { pour la population active) }\end{array}$ \\
\hline 1. Agriculteurs exploitants & 10. Agriculteurs exploitants \\
\hline $\begin{array}{l}\text { 2. Artisans, commerçants, } \\
\text { chefs d'entreprise }\end{array}$ & $\begin{array}{l}\text { 21. Artisans } \\
\text { 22. Commerçants et assimilés } \\
\text { 23. Chefs d'entreprise de } 10 \text { salariés ou plus }\end{array}$ \\
\hline $\begin{array}{l}\text { 3. Cadres et professions } \\
\text { intellectuelles supérieures }\end{array}$ & $\begin{array}{l}\text { 31. Professions libérales et assimilées } \\
\text { 32. Cadres de la fonction publique, professions intellectuelles et artistiques } \\
\text { (dont Professeurs et professions scientifiques) } \\
\text { 36. Cadres d'entreprise }\end{array}$ \\
\hline $\begin{array}{l}\text { 4. Professions } \\
\text { intermédiaires }\end{array}$ & $\begin{array}{l}\text { 41. Professions intermédiaires de l'enseignement, de la santé, de la fonction } \\
\text { publique et assimilés (dont Professeurs des écoles, instituteurs et assimilés) } \\
\text { 46. Professions intermédiaires administratives et commerciales des entreprises } \\
\text { 47. Techniciens } \\
\text { 48. Contremaîtres }\end{array}$ \\
\hline 5. Employés & $\begin{array}{l}\text { 51. Employés de la fonction publique } \\
\text { 54. Employés administratifs d'entreprise } \\
\text { 55. Employés de commerce } \\
\text { 56. Personnels des services directs aux particuliers }\end{array}$ \\
\hline 6. Ouvriers & $\begin{array}{l}\text { 61. Ouvriers qualifiés } \\
\text { 66. Ouvriers non qualifiés } \\
\text { 69. Ouvriers agricoles }\end{array}$ \\
\hline
\end{tabular}

Tableau 2. Le regroupement des PCS opéré par la DEPP

\begin{tabular}{|c|c|}
\hline Catégorie DEPP & PCS (en 18 postes) correspondantes \\
\hline Favorisée A & $\begin{array}{l}\text { 23. Chefs d'entreprise de } 10 \text { salariés ou plus } \\
\text { 31. Professions libérales et assimilées } \\
\text { 32. Cadres de la fonction publique, professions intellectuelles et artistiques } \\
\text { (dont Professeurs et professions scientifiques) } \\
\text { 36. Cadres d'entreprise + Professeurs des écoles, instituteurs et assimilés }\end{array}$ \\
\hline Favorisée B & $\begin{array}{l}\text { 41. Professions intermédiaires de l'enseignement, de la santé, de la fonction } \\
\text { publique et assimilés (sans les Professeurs des écoles, instituteurs et assimilés) } \\
\text { 46. Professions intermédiaires administratives et commerciales des entreprises } \\
\text { 47. Techniciens } \\
\text { 48. Contremaîtres }\end{array}$ \\
\hline Moyenne & $\begin{array}{l}\text { 10. Agriculteurs } \\
\text { 21. Artisans } \\
\text { 22. Commerçants et assimilés } \\
\text { 51. Employés de la fonction publique } \\
\text { 54. Employés administratifs d'entreprise } \\
\text { 55. Employés de commerce } \\
\text { 56. Personnels des services directs aux particuliers }\end{array}$ \\
\hline Défavorisée & $\begin{array}{l}\text { 61. Ouvriers qualifiés } \\
\text { 66. Ouvriers non qualifiés } \\
\text { 69. Ouvriers agricoles }\end{array}$ \\
\hline
\end{tabular}

